\title{
BMJ Open Understanding data and information needs for palliative cancer care to inform digital health intervention development in Nigeria, Uganda and Zimbabwe: protocol for a multicountry qualitative study
}

Kehinde Okunade, ${ }^{1}$ Kennedy Bashan Nkhoma, ${ }^{2}$ Omolola Salako, ${ }^{3}$ David Akeju, ${ }^{4}$ Bassey Ebenso (D) , ${ }^{5}$ Eve Namisango, ${ }^{6}$ Olaitan Soyannwo, ${ }^{7}$ Elizabeth Namukwaya, ${ }^{8}$ Adlight Dandadzi, ${ }^{9}$ Elizabeth Nabirye ${ }^{8}$ Lovemore Mupaza, ${ }^{10}$ Emmanuel Luyirika, ${ }^{6}$ Henry Ddungu, ${ }^{11}$ Z Mike Chirenje, ${ }^{9}$ Michael I Bennett, ${ }^{12}$ Richard Harding (D) , Matthew J Allsop ${ }^{12}$

To cite: Okunade K, Bashan Nkhoma K, Salako 0, et al. Understanding data and information needs for palliative cancer care to inform digital health intervention development in Nigeria, Uganda and Zimbabwe: protocol for a multicountry qualitative study. BMJ Open 2019;9:e032166. doi:10.1136/ bmjopen-2019-032166

- Prepublication history and additional material for this paper are available online. To view these files, please visit the journal online (http://dx.doi. org/10.1136/bmjopen-2019032166).

Received 06 June 2019 Revised 08 0ctober 2019 Accepted 09 0ctober 2019

Check for updates

(c) Author(s) (or their employer(s)) 2019. Re-use permitted under CC BY. Published by BMJ.

For numbered affiliations see end of article.

Correspondence to Dr Matthew J Allsop; m.j.allsop@leeds.ac.uk

\section{ABSTRACT}

Introduction Palliative care is a clinically and costeffective component of cancer services in sub-Saharan Africa (SSA). Despite the significant need for palliative cancer care in SSA, coverage remains inadequate. The exploration of digital health approaches could support increases in the quality and reach of palliative cancer care services in SSA. However, there is currently a lack of any theoretical underpinning or data to understand stakeholder drivers for digital health components in this context. This project addresses this gap through engaging with key stakeholders to determine data and information needs that could be supported through digital health interventions. Methods and analysis This is a multicountry, crosssectional, qualitative study conducted in Nigeria, Uganda and Zimbabwe. In-depth interviews will be conducted in patients with advanced cancer $(n=20)$, caregivers $(n=15)$, health professionals $(n=20)$ and policy-makers $(n=10)$ in each of the three participating countries. Data from a total of 195 interviews will transcribed verbatim and translated into English before being imported into NVivo software for deductive framework analysis. The analysis will seek to understand the acceptability and define mechanisms of patient-level data capture and usage via digital technologies.

Ethics and dissemination Ethics approvals have been obtained from the Institutional Review Boards of University of Leeds (Ref: MREC 18-032), Research Council of Zimbabwe (Ref: 03507), Medical Research Council of Zimbabwe (Ref: MRCZ/A/2421), Uganda Cancer Institute (Ref: 19-2018), Uganda National Council of Science and Technology (Ref: HS325ES) and College of Medicine University of Lagos (Ref: HREC/15/04/2015). The project seeks to determine optimal mechanisms for the design and development of subsequent digital health interventions to support development, access to, and delivery of palliative cancer care in SSA. Dissemination of these findings will occur through newsletters and press releases,
Strengths and limitations of this study

This study is the first to identify preferences of stakeholders involved in palliative cancer care provision in sub-Saharan Africa to inform digital health approaches.

- All aspects of the proposed study have been coproduced with experts in palliative care delivery and research in Nigeria, Uganda and Zimbabwe.

- This multicountry study will generate a logic model to target digital health approaches for palliative care that could have relevance across the sub-Saharan Africa region.

- This study restricts its focus to palliative cancer care so its relevance to other palliative conditions may be limited.

- The findings may not be reproducible beyond the three participating countries.

conference presentations, peer-reviewed journals and social media.

Trial registration number ISRCTN15727711

\section{INTRODUCTION}

Due to late-stage clinical presentation, limited funding and restricted access to curative therapies, about $80 \%$ of cancers on the continent are incurable at the time of detection and diagnosis. ${ }^{1}$ In 2018, there were over 770000 new cancer cases and 514000 cancer-related deaths across Eastern, Middle, Southern and Western Africa. ${ }^{2}$ These figures are projected to continue to rise (up to 1.28 million new cases and 970000 deaths) by 2030, with subsequent international and regional 
political declarations constituting a new global noncommunicable disease agenda. ${ }^{3}$ Increasing incidence is attributed to factors that include ageing, high residual burden of infectious agents (HIV/AIDS, human papillomavirus, hepatitis B virus) and lifestyle factors in subSaharan Africa (SSA). ${ }^{4}$ Our analysis reveals that by 2060 , an estimated 16 million people with cancer will die annually with serious health-related suffering, a $109 \%$ increase between 2016 and 2060, with the fastest rise occurring in low-income countries ( $400 \%$ increase).$^{5}$

Palliative care (PC) - the prevention and relief of physical, emotional, social or spiritual suffering associated with any chronic or life-threatening illness, from the point of diagnosis-is a vital and fundamental component of the basic and essential services within universal health coverage (UHC) ${ }^{6}$ It is also a realistic response to support equitable, accessible and cost-effective interventions for cancer care in SSA. Independent of cancer prevention and treatment efforts in the region, PC remains a critical and essential component of care, with proven effectiveness and cost-effectiveness. ${ }^{78}$ There have been enormous strides made in the development of PC services in the SSA region, ${ }^{910}$ but there remains a need for significant expansion of provision to meet demand. Current provision of PC services is limited to 24 of 48 countries, up from only five in 2004, with only less than $5 \%$ of people who need $\mathrm{PC}$ being able to access services in the region. ${ }^{10}$

A major challenge to developing palliative cancer care across the African region is the lack of local evidence to ensure practice is evidence-based and replicable and reflects the needs of the population served. ${ }^{6}$ Evidence to date has revealed that patients with advanced cancer in SSA have a high burden of physical and psychological symptoms, would prefer to have full information and better communication around their needs and care options, experience spiritual distress, their family caregivers face compounded poverty and psychological distress. ${ }^{11-18}$ It is essential to create channels for gathering patient-level data as an indicator of quality as well as to inform clinical practice and audit. Furthermore, understanding how emerging services are supporting patients with advanced cancer through assessing experiences and outcomes is a priority for PC development in the region. ${ }^{19}$ This can be achieved through the use of validated, context-specific tools for measuring outcomes for PC patients and their caregivers in SSA. ${ }^{19} 20$ We have therefore developed a valid patient-reported outcome measure (PROM) for advanced disease in SSA to capture the core concerns of patients and families. ${ }^{21-23}$ Capturing these data can enable development and adaptation of services to ensure they can respond to the specific needs of patients with cancer.

This protocol describes a study to explore how technology-based approaches could capture patient-level data from patients with advanced cancer that has utility across the health system. Previous work by our team has highlighted the potential of digital health to facilitate the collection, sharing and use of patient-level data. For example, we know that mobile phones are frequently used in multiple ways as part of PC service provision in the African region and that development of approaches that capitalise on mobile phones is a high priority for providers. ${ }^{24}$ In SSA, mobile phone services are available to a larger portion of the population than many basic services (such as sanitation and financial services). Approaches using digital health can benefit from the widespread access and low cost of mobile phone devices in the region and have shown improved chronic disease management, ${ }^{24}$ patient behavioural change and health systems strengthening, ${ }^{25}$ reduced costs of patient monitoring, improved adherence and better communication. These benefits are greatest in rural areas. ${ }^{26}$ Furthermore, such patient to provider telemedicine has recently been recommended by the WHO as an approach that can support health systems strengthening. ${ }^{27}$

In recent years, there has been exploratory research and development of digital health approaches in PC services in SSA. ${ }^{28-30}$ However, this project seeks to address the lack of a theoretical underpinning to interventions using digital health components in this context. Our project will undertake engagement with key stakeholders (patient, caregivers, health professionals and policymakers) across the health system to define the optimal mechanisms through which patient-level data, captured via digital health approaches, can be integrated into palliative cancer care delivery and improvement.

\section{RESEARCH OBJECTIVES AND QUESTIONS}

The study aims to answer the question, what are the optimal mechanisms through which patient-level data, captured via digital health, can be used in the development and delivery of palliative cancer care in SSA?

The specific objectives of the project are to:

1. Establish a consortium of academic researchers (from the UK, Nigeria, Uganda and Zimbabwe), service user advocates, non-governmental organisations, PC providers, policy-makers and digital health development and implementation experts to catalyse digital health research and generate evidence that can guide palliative cancer care development across SSA.

2. Understand the acceptability and optimal implementation of patient-level data collection (eg, PROMs and patient-reported experience measures) using digital health approaches in Uganda, Nigeria and Zimbabwe through patient and caregiver engagement.

3. Determine information needs and pathways for leveraging evidence generated from digital health approaches in service development in Uganda, Nigeria and Zimbabwe through health professional and service manager engagement.

4. Determine information needs and pathways for leveraging evidence generated from digital health approaches in policy-making in Uganda, Nigeria and Zimbabwe through policy-maker engagement. 
5. Define the mechanisms for the implementation of digital health approaches to support development of palliative cancer care in SSA.

6. Develop a theoretically informed logic model for implementing digital health approaches to improve PC in SSA.

\section{METHODS AND ANALYSIS}

Through partnership with the African Palliative Care Association (APCA), the University of Leeds have assessed the use and priorities of digital health approaches in $\mathrm{PC}$ services in the African region. ${ }^{28}$ Furthermore, King's College London, working with APCA, have been pioneering patient-level data collection in PC in SSA and developing PROMs ${ }^{19}{ }^{20}$; simple checklists of symptoms and concerns that are widely adopted and enable staff, patients and families to identify main concerns and prevent suffering, maintain people at home, support families and optimise function. PROMs for people with serious incurable illness can improve care and patient well-being. This project will enable integration of these research initiatives, determining the architecture of digital technologies to facilitate uptake and utilisation of evidence-based approaches such as PROMs. This will take forward the science of digital health in this neglected field, enabling a logic model to be developed for subsequent evaluation and implementation.

\section{PC development in participating countries}

The current development of PC for each participating country is summarised in table 1.

In recent years, policy reports of PC from participating study countries have commonly identified priority focus areas of improving access to pain medications, improving awareness by health professionals of the value and role of PC in supporting patients, and integration of PC with the existing public health system. A summary of policy documents and their key findings for each country is provided in online supplementary appendix A.

\section{Study design}

This study will adopt a multicountry, cross-sectional, exploratory study using qualitative methods. In-depth interviews with patients, caregivers, health professionals and policy-makers will be used to understand the acceptability and define mechanisms of patient-level data capture and usage. The interpretation of the findings will define optimal mechanisms through which patient-level data, captured via digital health, can be used in the development, delivery and improvement of palliative cancer care in SSA. Alongside the planned research activities, a consortium focused on digital technology for palliative cancer care will be formed. This will include researchers from SSA and the UK, alongside key stakeholders in each of the participating countries (ie, relevant policy representatives from ministries of health, civil society, patient advocates and digital health specialists) to understand: (1) current digital health development in Uganda,
Nigeria and Zimbabwe; (2) opportunities for capacity development around digital health in palliative cancer care and (3) routes to uptake and translation of findings from planned research activities.

\section{Study participants}

The study participants will be adults living with advanced cancer, their caregivers, their health professionals and policy-makers with a focus on cancer, non-communicable diseases and/or technology. Patients will include adults with advanced cancer receiving PC. Caregivers will include those supporting palliative cancer patients receiving care from recruiting facilities. Health professionals will be drawn from the clinical teams associated with study partner institutes alongside related services delivered in the locality. Policy-makers will comprise district or national level policy-makers working within cancer, noncommunicable diseases or digital health. Table 2 outlines the inclusion/exclusion criteria, sampling characteristics and sample size for the four stakeholder groups.

\section{Data collection and management \\ Patients}

Clinical staff at recruiting facilities will be responsible for identifying participants. These staff will be asked to verify that patients are aware of their PC diagnosis. This will be through review of clinical records and discussion with the participant's health professionals by the research assistant. Patients who are deemed ethically inappropriate by members of the clinical team, for example, where death is imminent, will not be approached. Participating patients will only be asked to participate in one face-to-face interview. The location of the interview will be decided by the patient or caregiver (ie, meeting either at their home, following a clinic appointment at a health facility, on the ward or at a neutral location, dependent on the patient's preference and the patient's clinical management at the time of the interview). Independent of the location chosen, a quiet and private room will be recommended to the patient for the interview. Arrangements will be made for such space at clinics and hospices ahead of interviews. A topic guide will be used to direct semistructured interviews with patients. The topic guide will explore current interaction with and access to PC services, their use of technology, the acceptability of using digital technology approaches to support interaction with health services and data collection, the clinical response anticipated from health services (with and without facilitation by digital health interventions) including perceptions of effective responses and alternative approaches to patientlevel data collection without mobile phones.

\section{Caregivers}

Caregivers of patients with advanced cancer will be identified and approached to participate by clinical staff at recruiting facilities. Caregivers will only be required to participate in one face-to-face interview. A topic guide will be used to direct semistructured interviews with 
Table 1 Summary of palliative care (PC) development in Nigeria, Uganda and Zimbabwe

\section{Country Summary of development}

Nigeria In Nigeria - the most populous country in SSA-PC is disparately spread in centres across the country. Historically, PC development in western Africa has been secondary to developments in the eastern and southern parts of the continent, and this continues to be the case. This is due mainly to the relatively low HIV prevalence, which meant that the region did not qualify to receive funding from the US President's Emergency Plan for AIDS Relief (PEPFAR) in the early 2000 s. $^{32}$ Widespread interest in PC development in Nigeria emanated from the pain and suffering witnessed by concerned health professionals in patients with advanced cancer. Early efforts by the Hospice Nigeria team included an advocacy visit by Anne Merriman (the founder of Hospice Africa Uganda in 1993) which yielded little result as there was no opioid analgesics in the country to manage the associated cancer pain. ${ }^{33}$ The current movement that has resulted in establishment of holistic PC services across Nigeria commenced in 1996 with the Ibadan 'Cancer pain group'. ${ }^{34}$ Concerted advocacy and other activities of the group facilitated importation of opioid analgesics by the Federal Ministry of Health for the management of severe pain by 2001 and morphine powder for oral morphine preparation by 2005 .

The first PC team was founded in 2003 at Ibadan, providing both hospital-based and home-based services, ${ }^{32}$ and the Hospice and PC Association of Nigeria was created in 2007. Members, most of whom received PC initiator's training at Hospice Africa, Uganda, function as individuals or teams across the six geopolitical areas of the country. Home-based PC continues as a very important form of PC delivery, with evidence of its benefits to patients and their families. ${ }^{35}$ Some aspects of PC are now included in the national guidelines for HIV and AIDS treatment and care in adolescents and adults (2012) as well as the national cancer control plan of the Federal Ministry of Health (2018-2022).

Current barriers to PC development include lack of government guidelines, poor knowledge about PC importance at all levelspolicy-makers, public, health professionals, lack of inclusion of PC in curricula of health professionals and in the national health budget and National Health Insurance Scheme, poor availability and accessibility of strong opioid analgesics for cancer pain management. However, since 2012, the 'Treat Pain project' and 'Pain Free Hospital Initiative' of the Federal Ministry of Health, Global Access to Pain Relief Initiative and American Cancer Society have improved the opioid situation ${ }^{36}{ }^{37}$ More can still be achieved through the training of more health professionals and change of attitude, increased funding and increased public awareness of the services. ${ }^{35}$ There has been some early context setting work suggesting willingness to explore the application of technology within PC services, such as the use of telemedicine. ${ }^{38}$

Uganda The Atlas of Palliative Care Development in Africa ranked Uganda highly in terms of palliative service development and its integration into the health system. ${ }^{39}$ Prior to this, The Economist Intelligence Unit's Quality of Death Index ${ }^{40}$ which is a 'measure of the quality of PC provided to adults in over 80 countries', ranked Uganda as 35th in the world. This index gives a general impression of the quality of life at the end of life and recognises continued development of PC services in Uganda. In terms for service delivery, Uganda has over 229 PC service outlets, for a population of 40 million people. These include hospices, homebased care and health facility-based outlets. The level of paediatric PC service development remains poor, with two paediatric PC service centres despite a large population of children and young people. ${ }^{1}$

In terms of the wider health system, Uganda has been progressing the presence and development of PC services. Uganda is building a critical mass for PC professionals with PC integrated into the medical and nursing and is a hub for training and education in PC, with diploma, certificate and degree courses in PC provided by universities, specialised PC institutions like Mildmay Uganda, and international organisations like African Palliative Care Association (APCA). In terms of health information systems, there are two national indicators of $\mathrm{PC}$ into the national electronic health information system: patients presenting with pain and those receiving morphine for pain management. With this advancement, to some extent, performance of PC can be monitored at the national level.

Access to pain medication is a crucial component of PC delivery in Uganda. The availability of the full analgesic ladder and palliative medicines remains poor, ${ }^{41}$ compromising care providers' ability to support management of symptoms and alleviate suffering associated with pain. However, Uganda has introduced prescribing across cadres other than doctors, such as PC nurse prescribers. Furthermore, the rolling out of local production of morphine within Hospice Africa Uganda led to significant reductions in costs associated with morphine procurement. ${ }^{42}$ This key opioid for moderate to severe pain is now provided free of charge and is supplied through the national supply chain mechanisms of the country. Although oral morphine is free, access still remains a challenge because of several factors such frequent stock outs, reluctance to prescribe morphine for fear of addictions as well as limited access to this medicine at primary healthcare level. ${ }^{29}$

PC is included in the national health strategic plan, and in the HIV care guidelines. A stand-alone PC policy was drafted in 2017 and is pending cabinet approval. It is envisioned that a stand-alone policy will be a strong pillar in giving a strategic direction to service development and evaluation to provide a comprehensive framework for the integration of PC services into the healthcare system. It will also be used as tool to advance financing for PC advocacy. 
Table 1 Continued

\begin{tabular}{|c|c|}
\hline ountry & Summary of development \\
\hline & 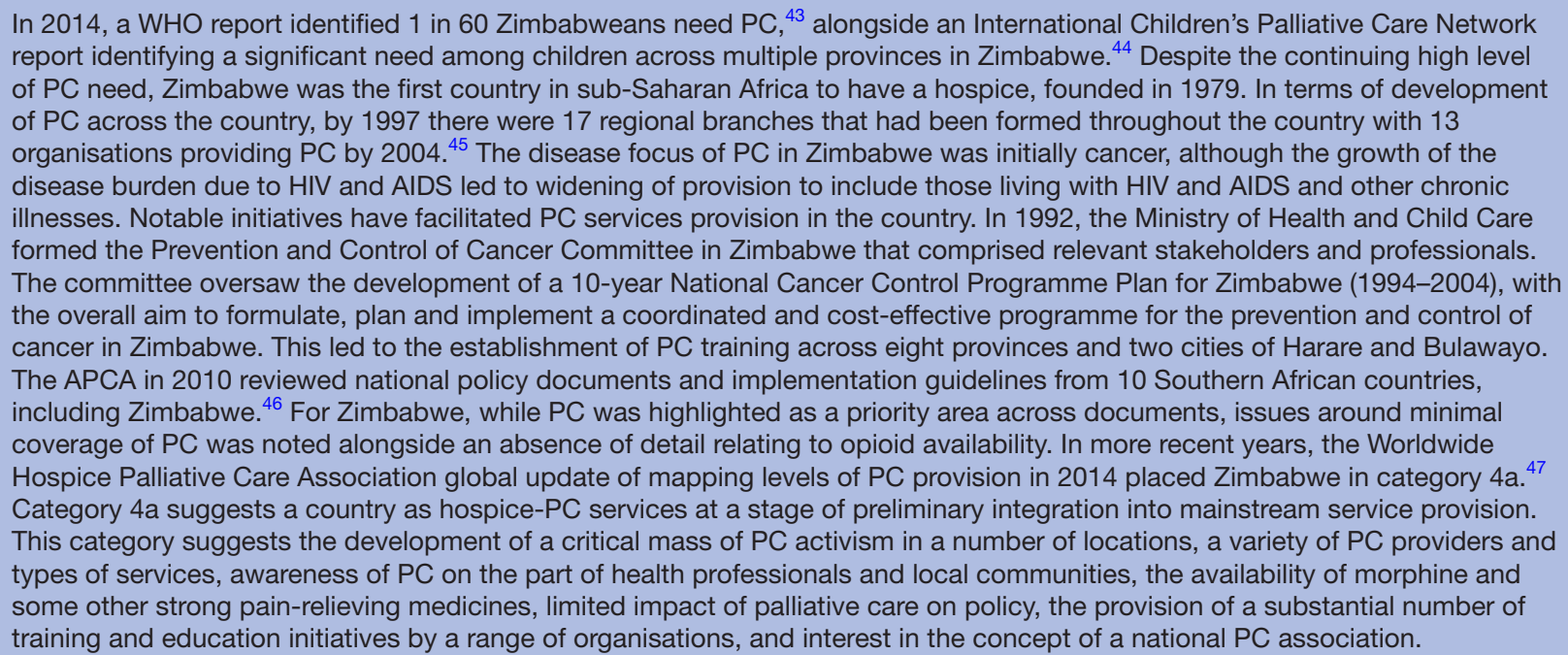 \\
\hline
\end{tabular}

caregivers. The themes addressed in the topic guide will align with the patient topic guide.

\section{Healthcare professionals}

Health professionals will be identified by clinical leads in each of the three countries. Through existing networks of PC providers in each of the countries, the clinical lead will approach health professionals to participate in the study. A topic guide will be used during health professional interviews. The topic guide aligns with stages of the data-use conceptual framework ${ }^{15}$ : data demand (eg, current availability, use and quality of data for clinical decision-making), data collection (eg, feasibility of digital technology approaches to patient-level data collection), data availability (eg, clinical triggers in management of patients with advanced cancer, capacity to respond to information, information needs to inform patient care) and data utilisation (eg, sharing and accessing data via digital health approaches, data reporting priorities to regional and national health authorities). Causal elements linked to organisational, technical and behavioural factors influencing data use will be explored for each stage.

\section{Policy-makers}

Policy-makers will be identified and approached by the APCA alongside academic and clinical teams in Nigeria, Uganda and Zimbabwe. A topic guide for key informant interviews will address access to and use of evidence to inform decision-making, seek comment on findings from a desktop review of existing policy on digital health to be conducted prior to key informant interviews, preferred mode and presentation of data, frequency of data reporting needed to inform decision-making on financing of PC services and on accelerating UHC.

Interviews across all recruitment sites in Uganda, Nigeria and Zimbabwe (outlined in online supplementary appendix B) will be undertaken by research assistants, supervised by the academic partners in Nigeria (KO), Uganda (ENamu) and Zimbabwe (MC). All interviews will be audio-recorded. Data security will be ensured through use of password-protected file sharing using the Microsoft OneDrive platform. Separate folders will be created for each country, with oversight from the lead institution, the University of Leeds. Only research team members will have access to the folder. All members of the project team will sign a data sharing agreement outlining explicit guidance regarding handling and management of research data that takes account of both the funders and national research council guidelines. On completion of this study, all electronic data on the OneDrive folder will be moved to an electronic archive for 5 years prior to being permanently destroyed. Deidentified research data deemed suitable for sharing will be hosted by Research Data Leeds, the institutional research data repository for the University of Leeds. All study participants will be assigned an identification code, which will be delinked from their identity at data entry point.

\section{Reflectivity}

The research team comprises experts in digital health intervention development (MJA, BE), qualitative research (DA, AD, ENamu), global PC provision (KBN, ENami, EL, $\mathrm{RH})$, monitoring and evaluation (LM), health services research in SSA (MC) alongside consultants in palliative medicine (ENamu, HD, OS, MIB) and oncologists (KO, OS). None of these researchers have any relationship with the patients or caregivers who will be approached to participate. However, a small number of the healthcare professionals or policy-maker participants may be known contacts.

\section{Patient and public involvement}

The rationale for this work arose from priorities identified through surveying a key stakeholder group, 
Table 2 Population and sampling criteria for the four stakeholder groups

Participant

group

Patient

$$
\text { (⿸丆口 }
$$

\section{Caregiver}

\section{Below 18 years}

\section{Inclusion criteria:}

- A primary caregiver/family caregiver of a patient with advanced cancer who is at least 18years of age

- Primary caregiver confirmed by the patient, including those who provide unpaid, informal provision of one or more physical, social, practical and emotional tasks. In terms of their relationship to the patient, they may be a friend, partner, ex-partner, sibling, parent, child or other blood or non-blood relative

- Both caregivers of participating and non-participating patients will be recruited into the study

\section{Exclusion criteria:}

- Below 18years of age

- Inadequate physical and mental health of a patient prior to recruiting (as deemed by the identifying clinician)

\begin{tabular}{|c|c|c|c|}
\hline $\begin{array}{l}\text { Health } \\
\text { professional }\end{array}$ & $\begin{array}{l}\text { Inclusion criteria: } \\
\text { Working with palliative care patients } \\
\text { At least } 6 \text { months working experience at recognised } \\
\text { palliative care facilities }\end{array}$ & $\begin{array}{l}\text { Role (doctor, clinical } \\
\text { officer, nurse, social } \\
\text { worker, psychologist, } \\
\text { pharmacist) } \\
\text { Typical work setting (ie, } \\
\text { community based, ward } \\
\text { based) }\end{array}$ & $\begin{array}{l}20 \text { purposely } \\
\text { selected health } \\
\text { professionals }\end{array}$ \\
\hline $\begin{array}{l}\text { Policy- } \\
\text { maker }\end{array}$ & $\begin{array}{l}\text { Inclusion criteria: } \\
\text { Representative from government ministry or national } \\
\text { association responsible for oversight and development } \\
\text { of healthcare in participating country }\end{array}$ & $\begin{array}{l}\text { Working at different levels } \\
\text { of the health system } \\
\text { (district, national) } \\
\text { Policy remit of their } \\
\text { post (cancer, non- } \\
\text { communicable diseases, } \\
\text { digital health) }\end{array}$ & $\begin{array}{l}\text { Up to } 10 \\
\text { purposely selected } \\
\text { policy-makers }\end{array}$ \\
\hline
\end{tabular}

healthcare providers delivering PC across SSA. ${ }^{24}$ Patients and caregivers were not involved in the design of the study. However, alongside research activities, the team will develop a consortium which will include patient advocates. This forum will be used to explore routes for communicating study findings to patient groups and help to establish potential routes for identifying patient and caregivers, or advocates, in subsequent projects.

\section{Target number} to recruit in each participating country

- 20 purposely selected patients

- Age

- Sex

- Cancer type

- Location at the time of interview (community based or ward based)

where the patient has not participated)

15 purposely selected caregivers

Age in study (ensuring representation of those where the patient whom they care for has participated, and those 
for deductive framework analysis. ${ }^{31}$ An initial charting of pseudonymised transcripts (KBN) will be developed through line-by-line coding. This initial code will then be applied to a random set of transcripts $(n=3)$ in each stakeholder group by three further members of the research team (Nigeria, Uganda, Zimbabwe), and the final framework agreed through discussion with the wider team. Once inconsistencies are resolved, the joint coding frame will be used to code all interviews across the participant group. Comparative analysis in the framework will enable us to identify common themes as well as country-specific and stakeholder group divergences. A model of the coding frame will be developed, and each theme and subtheme given a definition to ensure internal consistency of each code. Illustrative codes will be reported for each theme, with the study ID code to demonstrate reporting from across the sample breadth. In the final project meeting we will hold a Theory of Change workshop to model the planned digital health pathway within its context, detailing processes, stakeholder roles, outcomes and intended impact.

\section{Ethical considerations}

Ethics approvals have been obtained from the Institutional Review Boards of University of Leeds (Ref: MREC 18-032), Research Council of Zimbabwe (Ref: 03507), Medical Research Council of Zimbabwe (Ref: MRCZ/A/2421), Uganda Cancer Institute (Ref: 19-2018), Uganda National Council of Science and Technology (Ref: HS325ES) and College of Medicine University of Lagos (Ref: HREC/15/04/2015). Ethical review undertaken by all project investigators ensured standard processes (dignity, autonomy, informed consent, confidentiality, anonymity, ability to adhere to protocol) and data security were considered in the protocol development. The project will be aligned with the Medical Research Council good research practice guidelines and H3Africa framework for conducting ethically responsible biomedical research. With reference to research on PC populations, a patient's condition should not preclude them from participation. Instead, additional strategies of harm minimisation have been developed collaboratively by the research team. Academic leads and coinvestigators will support protocol adaptation for local cultural appropriateness (eg, modifying recruitment accounting for cancer-related stigma). Researchers will routinely contact clinical teams to check the health status of a patient prior to contact for research activities. In terms of data governance, project documentation and deidentified data for joint analysis will be shared via a secure IT infrastructure hosted by the University of Leeds. Voluntary and informed participation, confidentiality and safety of participants will constitute key principles of researcherrespondent interaction. Written consent or a thumb print will be obtained from patients, caregivers, healthcare professionals and policy-makers prior to their enrolment in the study.

\section{Dissemination}

We will provide an understanding of the mechanisms by which digital health approaches can facilitate evidence generation and use, ensuring optimal implementation with clear pathways for integrating captured data into existing health system functions. On completion of planned research activities, we will have:

1. Instigated an active, interdisciplinary consortium focused on technology-based approaches to developing palliative cancer care in SSA.

2. Defined mechanisms for optimal implementation of digital health interventions to support PC service development in SSA.

3. Provided a list of factors to target with digital health approaches with accompanying programme theory.

4. Obtained essential preliminary data needed to inform future research in digital health technology development for PC in SSA.

5. Developed a logic model for implementation of digital health to improve advanced cancer care in SSA.

6. Strengthened capacity for research, innovation and knowledge exchange in partner institutions and identified future capacity building needs.

Undertaking primary research with patients, caregivers, health professionals and policy-makers is essential to guide digital health approaches for palliative cancer care in SSA. A number of activities will be undertaking to support dissemination of project findings. These will include: (1) developing newsletters and press releases to communicate key project findings to the general public, (2) developing a dedicated website for the study where results will be publicly accessible by national and international policy-makers, practitioners and academics, (3) delivering presentations at local and national conferences in participating countries, alongside presentation at international conferences, (4) publishing articles in peer-reviewed journals and (5) social media through research team member and institutional accounts. Participants will be anonymised in any dissemination activities. Only pseudonymised, non-identifiable characteristics and quotes will be used in dissemination.

\section{CONCLUSION}

This paper reports the protocol for a cross-sectional study with qualitative methods aimed at understanding the optimal mechanisms through which patient-level data, captured via digital health, can be used in the development, access and delivery of quality palliative cancer care in Uganda, Nigeria and Zimbabwe. The findings of the study will be device agnostic, providing a theoretical framework that can be used to inform a wide range of digital health intervention development and implementation. Without this research there is a risk of digital health intervention development for PC occurring in silos across SSA that do not take account of the multiple uses and value of data for stakeholders across the wider health system. Mapping information and data needs 
across PC services will also create multiple opportunities for research. This includes subsequent piloting and evaluation of digital health interventions and validation of the data they capture, development of patient-focused digital health interventions such as information provision and self-management support, and exploration of the influence of factors such as gender, intersectionality, disability and cancer type on utilisation and engagement with digital health approaches. Subsequent development of digital health approaches for PC in SSA, gathering patient-level data and facilitating patient-provider communication, could lead to multiple benefits for patients and caregivers (reduced costs associated with time and travel to facilities, extend coverage and reach of services such as rural areas with mobile connectivity), health professionals (ability to identify and respond to specific and rising demand from patients with cancer) and policy-makers (receiving appropriate and timely data to inform service planning, guide integration of PC with wider healthcare delivery and contribute to strengthening of national digital health systems). There is an added imperative to understand how best to use digital health technologies for those receiving and providing care for advanced disease in SSA. There is currently a lack of evidence on the preferences of patients with advanced disease, their caregivers and their health professionals. Understanding these preferences is crucial to inform digital health intervention development, such as modelling interventions around the preferences of the timing and delivery of digital health approaches in PC.

\author{
Author affiliations \\ ${ }^{1}$ College of Medicine, University of Lagos, Lagos, Nigeria \\ ${ }^{2}$ Florence Nightingale Faculty of Nursing Midwifery and Palliative Care, Cicely \\ Saunders Institute, King's College London, London, UK \\ ${ }^{3}$ Department of Radiation Oncology, Lagos University Teaching Hospital, Lagos, \\ Nigeria \\ ${ }^{4}$ Department of Sociology, University of Lagos, Lagos, Nigeria \\ ${ }^{5}$ Nuffield Centre for International Health and Development, Leeds Institute of Health \\ Sciences, University of Leeds, Leeds, UK \\ ${ }^{6}$ African Palliative Care Association, Kampala, Uganda \\ ${ }^{7}$ Centre for Palliative Care, University College Hospital, Ibadan, Nigeria \\ ${ }^{8}$ Department of Internal Medicine, Makerere University, Kampala, Uganda \\ ${ }^{9}$ Clinical Trials Research Centre, College of Health Sciences, University of Zimbabwe, \\ Harare, Zimbabwe \\ ${ }^{10}$ Island Hospice and Healthcare, Harare, Zimbabwe \\ ${ }^{11}$ Uganda Cancer Institute, Kampala, Uganda \\ ${ }^{12}$ Academic Unit of Palliative Care, Leeds Institute of Health Sciences, University of \\ Leeds, Leeds, UK
}

\section{Twitter Bassey Ebenso @B_Ebenso and Richard Harding @RHardingCSI}

Acknowledgements We thank Olasupo Oyedepo, Director of the African Alliance of Digital Health Networks and Project Director at ICT4HEALTH Project for guidance on the development and implementation of this project.

Contributors MJA conceived the study; MJA, BE, RH, ENami, ENamu and $\mathrm{MC}$ contributed to the development of the study design and final protocols for sample selection, observations and interviews; KO, MJA, OS and ENami developed a draft of the manuscript; All authors contributed to writing the manuscript.

Funding This work was supported by the Medical Research Council (grant no MR/ S014535/1) and Research England Quality-Related Global Challenges Research Fund (QR GCRF) through the University of Leeds.

Competing interests None declared.
Patient consent for publication Not required.

Ethics approval Ethics approvals have been obtained from the Institutional Review Boards of University of Leeds (Ref: MREC 18-032), Research Council of Zimbabwe (Ref: 03507), Medical Research Council of Zimbabwe (Ref: MRCZ/A/2421), Uganda Cancer Institute (Ref: 19-2018), Uganda National Council of Science and Technology (Ref: HS325ES) and College of Medicine University of Lagos (Ref: HREC/15/04/2015).

Provenance and peer review Not commissioned; externally peer reviewed.

Data availability statement No additional data available.

Open access This is an open access article distributed in accordance with the Creative Commons Attribution 4.0 Unported (CC BY 4.0) license, which permits others to copy, redistribute, remix, transform and build upon this work for any purpose, provided the original work is properly cited, a link to the licence is given, and indication of whether changes were made. See: https://creativecommons.org/ licenses/by/4.0/.

\section{ORCID iDs}

Bassey Ebenso http://orcid.org/0000-0003-4147-0968

Richard Harding http://orcid.org/0000-0001-9653-8689

\section{REFERENCES}

1 Ferlay J, Soerjomataram I, Dikshit R, et al. Cancer incidence and mortality worldwide: sources, methods and major patterns in GLOBOCAN 2012. Int J Cancer 2015;136:E359-86.

2 International Agenct for Research on Cancer. Globocan: All cancers. France: International Agenct for Research on Cancer, 2018.

3 Powell RA, Ali Z, Luyirika E, et al. Out of the shadows: noncommunicable diseases and palliative care in Africa. BMJ Support Palliat Care 2017;7:128-32.

4 Jemal A, Vineis P, Bray F. The cancer atlas. Atlanta, Georgia: American Cancer Society, 2016.

5 Sleeman KE, de Brito M, Etkind S, et al. The escalating global burden of serious health-related suffering: projections to 2060 by world regions, age groups, and health conditions. Lancet Glob Health. In Press 2019;7:e883-92.

6 Knaul FM, Farmer PE, Krakauer EL, et al. Alleviating the access abyss in palliative care and pain relief-an imperative of universal health coverage: the Lancet Commission report. The Lancet 2018;391:1391-454.

7 Gomes B, Calanzani N, Curiale V, et al. Effectiveness and costeffectiveness of home palliative care services for adults with advanced illness and their caregivers. Cochrane Database Syst Rev 2013;36:CD007760.

8 Smith S, Brick A, O'Hara S, et al. Evidence on the cost and costeffectiveness of palliative care: a literature review. Palliat Med 2014;28:130-50.

9 Fraser BA, Powell RA, Mwangi-Powell FN, et al. Palliative care development in Africa: lessons from Uganda and Kenya. $J$ Glob Oncol 2018;4:1-10.

10 Downing J, Grant L, Leng M, et al. Understanding models of palliative care delivery in sub-Saharan Africa: learning from programs in Kenya and Malawi. J Pain Symptom Manage 2015;50:362-70.

11 Beynon T, Radcliffe E, Child F, et al. What are the supportive and palliative care needs of patients with cutaneous T-cell lymphoma and their caregivers? A systematic review of the evidence. $\mathrm{Br} J$ Dermatol 2014;170:599-608.

12 Harding R, Selman L, Agupio G, et al. The prevalence and burden of symptoms amongst cancer patients attending palliative care in two African countries. Eur J Cancer 2011;47:51-6.

13 Selman L, Higginson IJ, Agupio G, et al. Meeting information needs of patients with incurable progressive disease and their families in South Africa and Uganda: multicentre qualitative study. BMJ 2009;338.

14 Selman LE, Higginson IJ, Agupio G, et al. Quality of life among patients receiving palliative care in South Africa and Uganda: a multicentred study. Health Qual Life Outcomes 2011;9:21.

15 Selman L, Harding R, Higginson IJ, et al. Spiritual wellbeing in subSaharan Africa: the meaning and prevalence of 'feeling at peace'. BMJ Support Palliat Care 2011;1.

16 Selman L, Siegert RJ, Higginson IJ, et al. The MVQOLI successfully captured quality of life in African palliative care: a factor analysis. $J$ Clin Epidemiol 2011;64:913-24.

17 Selman L, Siegert RJ, Higginson IJ, et al. The "Spirit 8" successfully captured spiritual well-being in African palliative care: factor and Rasch analysis. J Clin Epidemiol 2012;65:434-43. 
18 Streid J, Harding R, Agupio G, et al. Stressors and resources of caregivers of patients with incurable progressive illness in subSaharan Africa. Qual Health Res 2014;24:317-28.

19 Bausewein C, Daveson BA, Currow DC, et al. EAPC White Paper on outcome measurement in palliative care: Improving practice, attaining outcomes and delivering quality services Recommendations from the European Association for Palliative Care (EAPC) Task Force on Outcome Measurement. Palliat Med 2016:30:6-22.

20 Harding R, Selman L, Agupio G, et al. Validation of a core outcome measure for palliative care in Africa: the apcA African palliative outcome scale. Health Qual Life Outcomes 2010;8:10

21 Blum D, Selman LE, Agupio G, et al. Self-report measurement of pain \& symptoms in palliative care patients: a comparison of verbal, visual and hand scoring methods in Sub-Saharan Africa. Health Qual Life Outcomes 2014;12:118.

22 Harding R, Selman L, Agupio G, et al. Validation of a core outcome measure for palliative care in Africa: the apcA African palliative outcome scale. Health Qual Life Outcomes 2010;8:10.

23 Harding R, Selman L, Simms VM, et al. How to analyze palliative care outcome data for patients in sub-Saharan Africa: an international, multicenter, factor analytic examination of the apcA African Pos. $J$ Pain Symptom Manage 2013;45:746-52.

24 Beratarrechea A, Lee AG, Willner JM, et al. The impact of mobile health interventions on chronic disease outcomes in developing countries: a systematic review. Telemed J E Health 2014;20:75-82.

25 Peiris D, Praveen D, Johnson C, et al. Use of mHealth systems and tools for non-communicable diseases in low- and middleincome countries: a systematic review. J Cardiovasc Trans/ Res 2014;7:677-91.

26 Betjeman TJ, Soghoian SE, Foran MP. mHealth in sub-Saharan Africa. Int J Telemed Appl 2013;2013:1-7.

27 World Health Organization. Recommendations on digital interventions for health system strengthening. Geneva, Switzerland: World Health Organization, 2019.

28 Allsop MJ, Namisango E, Powell RA. A survey of mobile phone use in the provision of palliative care services in the African region and priorities for future development. J Telemed Telecare 2019;25:230-40.

29 Namisango E, Ntege C, Luyirika EBK, et al. Strengthening pharmaceutical systems for palliative care services in resource limited settings: piloting a mHealth application across a rural and urban setting in Uganda. BMC Palliat Care 2016;15:20.

30 Allsop MJ, Powell RA, Namisango E. The state of mHealth development and use by palliative care services in sub-Saharan Africa: a systematic review of the literature. BMJ Support Palliat Care 2018;8:155-63.
31 Gale NK, Heath G, Cameron E, et al. Using the framework method for the analysis of qualitative data in multi-disciplinary health research. BMC Med Res Methodol 2013;13:117.

32 Ajayi IO, Iken O, Powell RA. Palliative care research in Western Africa. Eur J Palliat Care 2014;21:45-7.

33 Merriman A. Audacity to Love: The Story of Hospice Africa. Ireland: Irish Hospice Foundation, 2010.

34 Soyannwo O. Hospice and palliative care development in West Africa sub region: a review. African J Anaesthesia and Intensive Care 2013;13:10-13.

35 Omoyeni N, Soyannwo O, Aikomo O, et al. Home-Based palliative care for adult cancer patients in Ibadan-a three year review. Ecancermedicalscience 2014;8:490.

36 O'Brien M, Mwangi-Powell F, Adewole IF, et al. Improving access to analgesic drugs for patients with cancer in sub-Saharan Africa. Lancet Oncol 2013;14:e176-82.

37 O'Brien M, Schwartz A, Plattner L. Treat the pain program. J Pain Symptom Manage 2018;55:S135-9.

38 van Gurp J, Soyannwo O, Odebunmi K, et al. Telemedicine's potential to support good dying in Nigeria: a qualitative study. PLoS One 2015;10:e0126820.

39 Rhee JY, Luyirika E, Namisango E, et al. APCA Atlas of Palliative Care in Africa. Texas, USA: IAHPC Press, 2017.

40 The Economist Intelligence Unit. The 2015 Quality of Death Index: Ranking palliative care across the world 201. London, UK: The Economist, 2015.

41 Harding R, Simms V, Penfold S, et al. Availability of essential drugs for managing HIV-related pain and symptoms within 120 PEPFARfunded health facilities in East Africa: a cross-sectional survey with onsite verification. Palliat Med 2014;28:293-301.

42 Merriman A, Harding R. Pain control in the African context: the Ugandan introduction of affordable morphine to relieve suffering at the end of life. Philos Ethics Humanit Med 2010;5:10

43 Khumalo T, Maasdorp V. The island hospice model of palliative care. Ecancermedicalscience 2016;10:654-54.

44 United Nations Children's FundConnor SR, Sisimayi C, eds. Assessment of the need for palliative care for children: three country report: South Africa, Kenya and Zimbabwe. United Nations Children's Fund, 2013.

45 Zimbabwe Ministry of Health and Child Care. The National palliative care policy. Harare, Zimbabwe: The Department of Epidemiology \& Disease Control Non-Communicable Diseases Unit, Zimbabwe Ministry of Health and Child Care, 2014.

46 African Palliative Care Association. Review of the status of palliative care in ten Southern African countries. Kampala, Uganda: African Palliative Care Association, 2016.

47 Connor SR, Bermedo M, eds. Worldwide Palliative Care Alliance, Global Atlas of Palliative Care at the End of Life. London: World Health Organization, 2014. 